\title{
Is mitral balloon valvuloplasty really innocent?: A case report of right ventricular rupture
}

\author{
(1) Hüseyin Sicim, (1) Ertan Demirdaş, (1) Hakan Kartal, (1) Gökhan Arslan, (1) Celalettin Günay, \\ (1) Bilgehan Savaş Öz
}

University of Health Sciences Turkey, Gülhane Faculty of Medicine, Department of Cardiovascular Surgery, Ankara, Turkey

\section{Date submitted:}

25.03.2020

Date accepted:

20.04.2020

Online publication date:

15.12.2020

\section{Corresponding Author:}

Hüseyin Sicim MD, University of Health Sciences Turkey, Gülhane Faculty of Medicine, Department of Cardiovascular Surgery, Ankara, Turkey

drhuseyinsicim@gmail.com

ORCID:

orcid.org/0000-0003-3430-3862

Presented in: 19. National Vascular and Endovascular Surgery Congress $10^{\text {th }}$ National Phlebology Congress 26-29 October 2019/Elexus Resort Hotel, Kyrenia, KKTC.

Keywords: Mitral balloon valvuloplasty, percutaneous, ventricular rupture

\begin{abstract}
Percutaneous mitral balloon valvuloplasty (PMBV) is the primary treatment modality in patients with mitral stenosis without contraindications and with appropriate valve anatomy. In the literature, the mortality rate associated with PMBV was reported to be $1 \%$. This process may cause rare and serious complications; such as pericardial effusion, acute mitral regurgitation, cardiac tamponade, and thrombus formation. In this case report, we present a 45 -year-old female patient who underwent emergency surgery because of right ventricular rupture during PMBV.
\end{abstract}

\section{Introduction}

Mitral balloon valvuloplasty is a method that has proven to be increasingly widespread and effective as a percutaneous intervention for mitral stenosis (1). Serious complications can occur during percutaneous cardiac catheterization, which may cause mortality and morbidity. Percutaneous mitral valvuloplasty is among the procedures that cause the highest rate of cardiac tamponade (2). In the literature, the frequency of developing cardiac tamponade during mitral balloon valvuloplasty has been found to be between $1 \%$ and $9 \%$ (3). Pericardiocentesis or emergency surgical intervention may be required due to cardiac tamponade. In this case report, we presented the successful treatment of right ventricular rupture, as an unprecedented complication during percutaneous mitral balloon valvuloplasty (PMBV).

\section{Presentation of Case}

A 45-year-female patient presented with symptoms of palpitation and shortness of breath since last twelve months. On her clinical examination, her pulse was $75 / \mathrm{min}$; rhythmic and regular, blood pressure was $120 / 80 \mathrm{mmHg}$. There was 
an opening snap, 3/8 diastolic murmur and 2/6 holosystolic murmur in cardiac auscultation. Blood tests were normal. Normal sinus rhythm was detected in electrocardiography. In 2D echocardiography, severe mitral stenosis was detected, and the mitral valve area was $1.3 \mathrm{~cm}^{2}$ with $\mathrm{PHT}$ and the valve gradient was $25 / 12 \mathrm{mmHg}$. Also, commissural calcification and moderate pulmonary hypertension $(65 \mathrm{~mm}$ of $\mathrm{Hg}$ ) were detected. The procedure was performed under sedation and with transesophageal echocardiography. Brockenbrough needle was used for transseptal puncture through the right femoral vein approach. Contrast-enhanced ventriculography was performed due to the development of bradycardia and hypotension during the procedure. The contrast was observed in the extracardiac area (Figure 1). The patient was taken to the operating room urgently. The patient underwent median sternotomy. After opening the pericardium, hemorrhagic fluid was evacuated. The catheter tip which ruptured the right ventricle was seen (Figure 2). A purse stitch was placed around the catheter and the catheter was withdrawn. The rupture was repaired with 4.0 prolene suture. Mitral valve replacement was performed after bleeding control was achieved. We used $25 \mathrm{~mm}$ St. Jude Medical mechanical prosthesis (St. Jude Medical, Inc., St. Paul, Minn.) with 4-0 pledget-supported Ti-Cron sutures (Davis \& Geck, Inc., Danbury, Conn.). The operation was terminated without complications. The patient was discharged from the hospital on the $7^{\text {th }}$ postoperative day.

\section{Discussion}

PMBV is a safe and effective treatment modality in patients with mitral stenosis, morphologically appropriate and symptomatic. The first successful PBMV was made in 1984 by Inoue (4). The Inoue technique can be widely accepted and used more widely for PBMV (5). General complications of PBMV are acute mitral regurgitation (MR), thromboembolism, and cardiac perforation. Perceptional intervention indication areas are expanding with experience. However, patients who are not eligible for percutaneous intervention are guided to surgical valve replacement or are treated medically in high surgical risk situations.

The most important complications of mitral balloon valvuloplasty procedure are death, shock, severe MR, systemic embolism, cardiac tamponade, emergency surgical need, and acute myocardial infarction. Minor complications are vasovagal reaction, prolonged hypotension, and arrhythmias requiring treatment. The incidence of all these complications is approximately $12 \%$ (6). Cardiac perforation followed by tamponade develops in approximately $4 \%$ of patients and often causes death during the procedure (6). Significant complications have been seen less frequently in recent years with processor experience and carefully administered septostomy.

In invasive cardiology, transthoracic or transesophageal echocardiography is often used to provide safety and control. It is essential for minimizing the complication rate in the procedure to be performed. Professional support is essential in these types of interventions, such as mitral balloon valvuloplasty, which are more sensitive, and complications are more serious. It is useful to follow the intracardiac catheter location, direction and complications such as perforation. It helps pinpoint exact location with real-time scanning (7). One of the most important issues to prevent complications during the procedure is that the

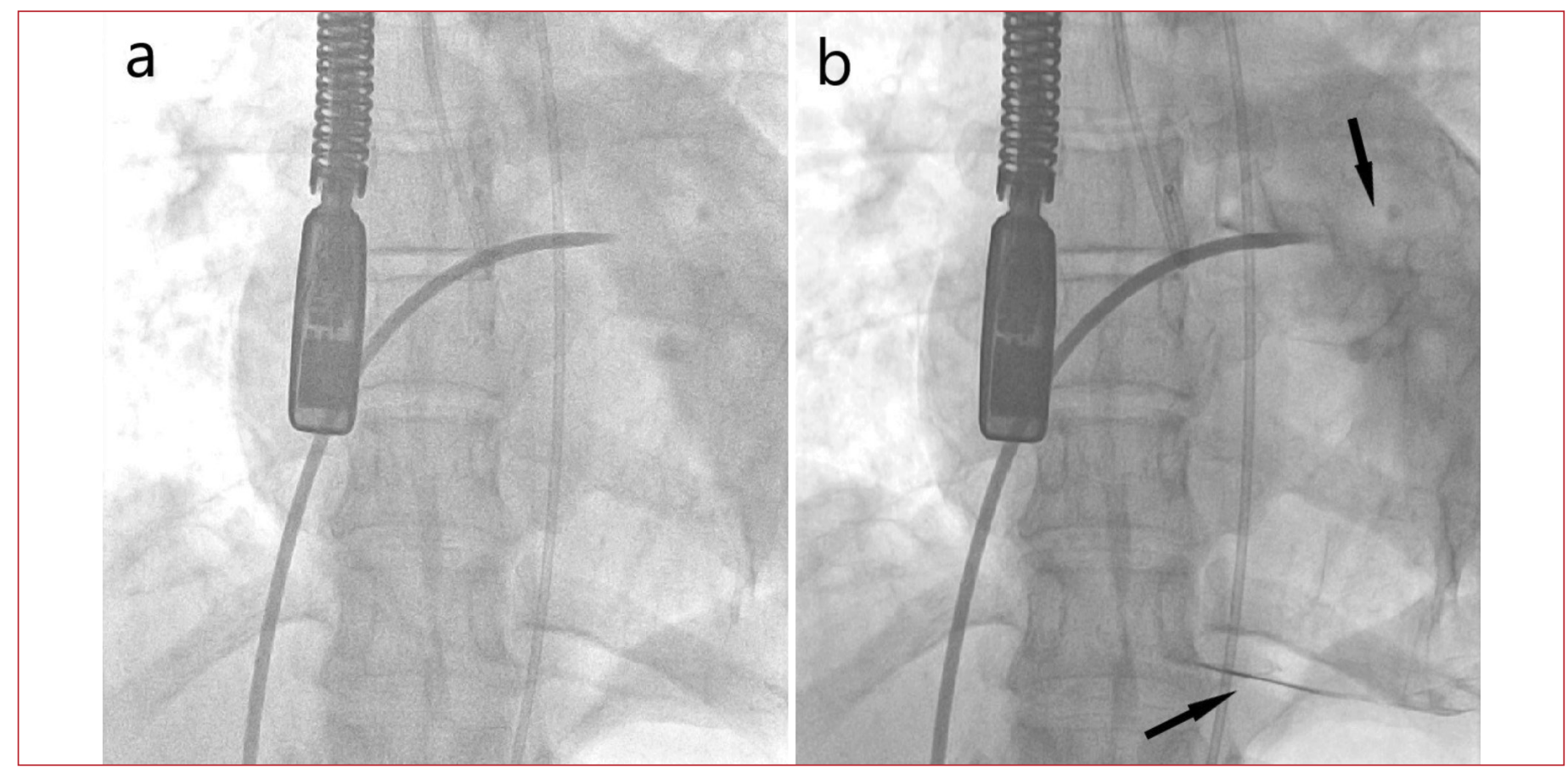

Figure 1. Contrast agent leaks to the extra cardiac area before (a) and after (b) contrast-enhanced ventriculography (arrows) 


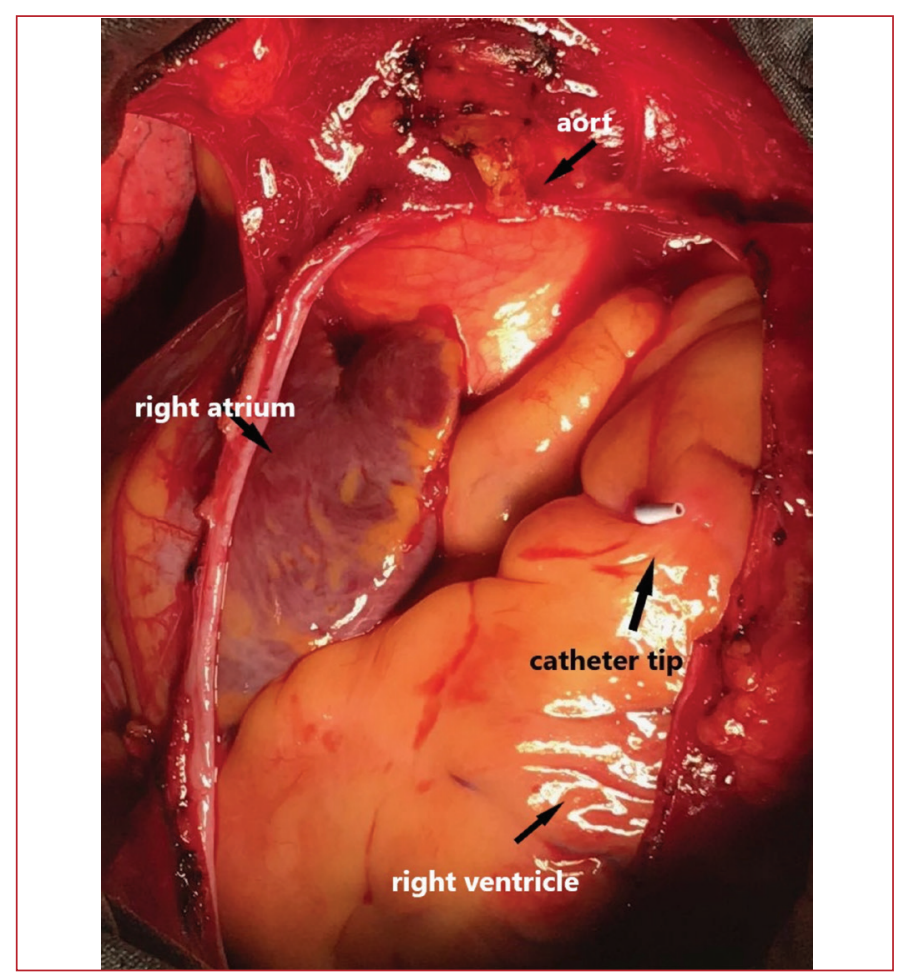

Figure 2. Intraoperative image of the catheter tip causing right ventricular rupture

doctor who performs echocardiography should have sufficient experience.

As shown in our case; mitral balloon valvuloplasty is widely used in patients with mitral stenosis and its never before seen and unexpected complications may occur. The surgical team should always be present against these complications with a high risk of mortality.

\section{Conclusion}

As a result of developing technology and minimally invasive methods, transseptal mitral commissurotomy is an effective and still safe method. Due to the low risk of complications after the procedure and its being minimally invasive for the patient, it continues to be preferred today. However, the most important issue to be considered is that the team should have sufficient experience and be able to perform emergency surgical intervention for possible mortal complications.

\section{Ethics}

Informed Consent: The patient gave an informed consent to publish the case report without revealing his identity, which was followed.

Peer-review: Externally peer-reviewed.

\section{Authorship Contributions}

Surgical and Medical Practices: H.S., E.D., Concept: H.K., G.A., Design: H.S., Data Collection or Processing: E.D., Analysis or Interpretation: C.G., Literature Search: B.S.Ö., Writing: H.S.

Conflict of Interest: No conflict of interest was declared by the authors.

Financial Disclosure: The authors declared that this study received no financial support.

\section{References}

1. Kamada M, Ohsaka K, Nagamine S, Kakihata H. Mitral valve replacement after percutaneous transluminal mitral commissurotomy. Jpn J Thorac Cardiovasc Surg. 2004;52:335-338.

2. Park SJ, Lee WK, Shim WH, Cho SY, Tahk SJ, Kim SS. Percutaneous mitral valvuloplasty using the double balloon technique: immediate results and determinant factors of increasing mitral regurgitation. Korean $\mathrm{J}$ Intern Med. 1991;6:51-57.

3. Raghul G, Gopalan R, Sundram SR, Arunkumar P, Tamilarasu K, Ramasamy P. Pericardial effusion as unexpected sequelae of postmitral balloon valvuloplasty. Heart India. 2016;4:104-107.

4. Prendergast BD, Shaw TR, lung B, Vahanian A, Northridge DB. Contemporary criteria for the selection of patients for percutaneous balloon mitral valvuloplasty. Heart. 2002;87:401-404.

5. Desabandhu V, Peringadan NG, Krishnan MN. Safety and efficacy of percutaneous balloon mitral valvotomy in severe mitral stenosis with moderate mitral regurgitation - A prospective study. Indian Heart J. 2016;68:783-787.

6. Fawzy ME. Mitral balloon valvuloplasty. J Saudi Heart Assoc. 2010;22:125-132.

7. Watanabe N. Editorial: Percutaneous mitral valvotomy: Balloon made in Japan still dominates the world. J Cardiol Cases. 2016;13:169-170. 